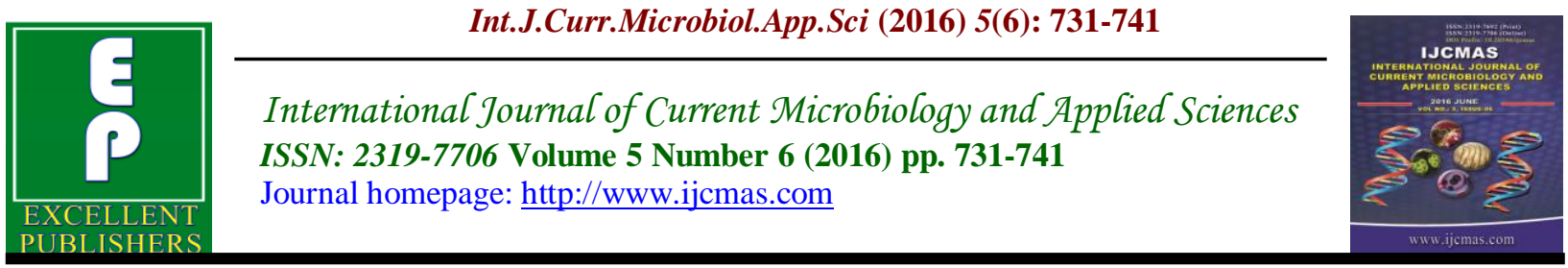

Review Article

http://dx.doi.org/10.20546/ijcmas.2016.506.079

\title{
Trichomoniasis-A Review
}

\author{
A. Hodiwala Bhesania ${ }^{1 *}$ and A. Narayankhedkar ${ }^{2}$ \\ ${ }^{1}$ Professor, Department of Microbiology, MGM Medical College and Hospital, Kamothe, \\ Navi Mumbai, India \\ ${ }^{2}$ Consultant Microbiologist, Dr Shahbazkar's Labortaory, Colaba, Mumbai, India \\ *Corresponding author
}

\author{
A B S T R A C T
}

Keywords

Trichomoniasis,

T.vaginalis, Morbidity.

\section{Article Info}

Accepted:

25 May 2016

Available Online:

10 June 2016
Trichomoniasis is a sexually transmissible infection that is capable of causing substantial morbidity. Trichomonas vaginalis is the only pathogenic species of the genus Trichomonas and has a worldwide distribution. The disease affects individuals in all racial groups and depending on the type of subpopulation studied prevalence rates vary between 0 and $65 \%$. In men, the organism can be found in the urethra, periurethral glands, prostate, epididymis, and in semen. In 2001, WHO estimated that 174 million cases occur worldwide annually, making T.vaginalis infection the most common non-viral STI. In the US, trichomoniasis affects two to three million women annually. Reports from China indicate that it accounts for more than $30 \%$ of gynaecologic inflammatory diseases causing female sterility. A Korean study done on patients complaining of vaginal symptoms found that $10.4 \%$ of these patients were infected by $T$. vaginalis. The prevalence of trichomoniasis at STD clinics varies from eight to $31 \%$. In KwaZulu-Natal, trichomoniasis is one of the most commonly reported STIs in females. A prevalence rate of $28 \%$ among STD clinic attendees was reported in 2004. It was found that the prevalence of trichomoniasis was lower in women attending antenatal clinics. Studies done, amongst pregnant women in South Africa show prevalence rates between 15 and 41.4\%.The prevalence of Trichomoniasis $2.8 \%-8.5 \%$ in India. These figures underestimate the real prevalence, since up to one half of all infections are asymptomatic and never diagnosed. Asymptomatic carriers can unknowingly transmit T.vaginalis via unprotected sexual contact. Therefore, asymptomatic infections are epidemiologically important. Hence it is important to diagnose this infection at the earliest to minimize the transmission amongst healthy population.

\section{Introduction}

Trichomoniasis is caused by the protozoan Trichomonas vaginalis.

\section{History of Trichomonas vaginalis}

Alfred Francois Donne (1836) Observed both the appearance and size of the protozoan which he referred to as 'Trichomonas'. Ehrenberg Protozoology pointed out that the protozoans human habitat is vaginal and therefore specifically named the protozoan Trichomonas vaginalis. Hesseltine (1942) Found that 13\% 
of female patients inoculated with $T$. vaginalis culture had developed trichomoniaisis. Lanceley \& McEntegart (1953) Inoculated five male volunteers with T.vaginalis culture, three of which developed urethritis.

\section{Morphology}

Trichomonas vaginalis is a prokaryotic organism with a eukaryotic nucleus. It belongs to the Sub-Kingdom Protozoa and Sub-Phylum Mastigophora, means "Flagella bearers". The family of Trichomonadidae consists of seven genera: Trichomonas, Pentatrichomonas, Tetratrichomonas, Pentatrichomonoides, Trichomitopsis, Pseudotrypanosoma and Cochlosoma. Trichomonas vaginalis is the largest of three species of the genus Trichomonas inhabiting man. The other two are Trichomonas hominis and Trichomonas tenax. Trichomonas vaginalis is characterized by having trophozoites or feeding stages only.

This means that no cystic, environmental resistant form exists. This explains the need for transmission through direct contact between mucosal surfaces.

The organism (as shown in figure below) is microscopic in size (approximately $10 \mu \mathrm{m}$ long and $7 \mu \mathrm{m}$ wide) and complex in structure, physiology and behavior. The oval trophozoite has a short undulating membrane, four flagella (filamentous outgrowths of the ectoplasm) and a clearly visible barb-like axostyle.

The axostyle may be used for surface attachment to vaginal epithelial cells (VECs) causing tissue damage. Trichomonas vaginalis also has a basic cytostome or gullet (mouth aperture) which helps to maintain the intraluminal position. In vitro, trichomonal cells appear ovoid or pearshaped whereas in the presence of VECs, they display an amoeboid appearance.

In culture; the dividing growth phase contains several oversized round forms either without flagella or with flagella equipped with a dividing nucleus or multiple nuclei. Trichomonas vaginalis is highly sensitive to both atmospheric oxygen and the lack of moisture and will therefore not survive for more than a few hours outside of the body.

\section{Reproduction and Life Cycle}

Trichomonas vaginalis multiplies by means of longitudinal binary fission. While the nuclear divisions in other protozoans are either mitotic or meiotic, trichomonads are an exception. They reproduce by a special type of closed mitosis called cryptopleuromitosis, a process similar to that of prokaryotic cells. The process of cryptopleuromitosis involves the development of spindle microtubules from two attractophores flanking the nucleus.

These spindle microtubules extend into the nucleus and attach to the kinetochores of the chromosomes. These spindle microtubules also elongates between the two attractophores ultimately separating the two daughter cells. Each daughter cell reconstructs by producing any missing organelles.

\section{Cell Function}

Trichomonas vaginalis lacks important eukaryotic organelles, such as mitochondria, peroxisomes and enzymes needed to conduct oxidative phosphorylation. Nutrients are obtained by both phagocytosis and transport through the cell membrane.

Trichomonas vaginalis produces adenosine 
triphosphate (ATP), by utilizing enzymes typically found in anaerobic bacteria. They do so by means of a unique, double membrane bound organelle called the hydrogenosome. These anaerobic mitochondria equivalents divide via fission and produce ATP via substrate phosphorylation. However, they differ from real mitochondria in that they do not contain genetic material and lack cytochromes. The controversy surrounding mitochondrial symbiosis and divergence of trichomonads from the main eukaryotic tree was resolved by a study showing the presence of VaIRS proteins and mitochondrial heat shock proteins in $T$. vaginalis. This study suggests that mitochondrial symbiosis had occurred first.

\section{Epidemiology}

Trichomoniasis is a sexually transmissible infection that is capable of causing substantial morbidity. Trichomonas vaginalis is the only pathogenic species of the genus Trichomonas and has a worldwide distribution. The disease affects individuals in all racial groups and depending on the type of subpopulation studied prevalence rates vary between 0 and $65 \%$. In men, the organism can be found in the urethra, periurethral glands, prostate, epididymis, and in semen. In 2001, WHO estimated that 174 million cases occur worldwide annually, making T.vaginalis infection the most common non-viral STI In the US, trichomoniasis affects two to three million women annually. Reports from China indicate that it accounts for more than $30 \%$ of gynaecologic inflammatory diseases causing female sterility. A Korean study done on patients complaining of vaginal symptoms found that $10.4 \%$ of these patients were infected by $T$. vaginalis. The prevalence of trichomoniasis at STD clinics varies from eight to $31 \%$. In KwaZulu-
Natal, trichomoniasis is one of the most commonly reported STIs in females. A prevalence rate of $28 \%$ among STD clinic attendees was reported in 2004. It was found that the prevalence of trichomoniasis was lower in women attending antenatal clinics. Studies done, amongst pregnant women in South Africa show prevalence rates between 15 and $41.4 \%$. The prevalence of Trichomoniasis $2.8 \%-8.5 \%$ in India. These figures underestimate the real prevalence, since up to one half of all infections are asymptomatic and never diagnosed. Asymptomatic carriers can unknowingly transmit T.vaginalis via unprotected sexual contact. Therefore, asymptomatic infections are epidemiologically important. Sexual intercourse is the most common route of transmission. Trichomonas vaginalis can survive for several hours outside the host; however, non-venereal transmission is extremely rare.

\section{Host Defence Mechanisms}

Although recurrent infections are common, the infection does not appear to confer immunity. However, immunity to $T$. vaginalis infection may be important in restricting the organism to the genito-urinary tract by stimulating non-specific responses. These responses include complement activation via the alternative pathway as well as neutrophil phagocytosis.

Infection caused in men is short-lived and this could be due to the presence of zinc in the prostatic fluid which has been shown to be cytotoxic to $T$. vaginalis, and therefore may be an inhibiting factor. Also, the reducing environment of the vagina is sufficient to activate proteinases involved in pathogenesis. However, the male genital tract is oxidative, thereby inhibiting such mechanisms. 
The ecology of the vaginal tract during the reproductive years proves highly significant in determining the severity of infection. Infection in the pre-menarchal girl and postmenopausal women is generally mild and transient as compared to women of the reproductive age. This is due to possible pathogenic factors, such as the vaginal $\mathrm{pH}$, iron concentrations in menstrual flow and estrogen fluctuations.

Dependability of the organism to iron concentrations in the secretions is apparent. When iron supply is low, it migrates deeper into the female genital tract in search of richer iron supply resulting in infection.

It is amazing how this organism survives dramatic environmental changes, such as the influx of host macromolecules, erythrocytes, serum constituents and $\mathrm{pH}$ changes. Research focusing on the initial events of infection is essential in understanding how the organism establishes infection.

\section{Pathogenesis}

Trichomonas vaginalis elaborates several mechanisms that contribute to pathogenesis during infection.

Being the most intensely studied trichomonad, these pathogenic mechanisms have not been clearly elucidated. Mechanisms utilized by this parasite include: interaction with the vaginal flora, immune evasion, recognition and binding to mucin, cytoadherence mediated by adhesins, haemolysis and the excretion of soluble factors, degrading all immunoglobulin subclasses and complement. The surface of the organism is a vital component of the complex host-parasite interaction. The fact that $T$. vaginalis is capable of long term survival in the adverse vaginal milieu demonstrates the highly evolved nature of this organism. Focusing on the initial stages of infection provides a clearer understanding of the pathogenesis of $T$. vaginalis. This in turn, could aid in developing new intervention strategies, better treatment, and better disease control.

\section{Clinical Manifestation}

Trichomonas vaginalis mainly infects the squamous epithelium of the female genital tract. This organism has previously been found in the urethra, fallopian tubes and the pelvis. Clinically trichomoniasis varies from an asymptomatic carrier state to overt vaginitis. This however, depends on the severity of the disease. One third of asymptomatic women display clinical trichomoniasis within six months. Acute trichomoniasis is characterized by diffuse vulvitis and vaginitis with pruritis, dyspareunia and dysuria. Small punctuate haemorrhagic spots often appear on the cervical mucosa, a situation termed the "strawberry cervix". Symptoms include a profuse,mucopurulent, frothy, malodorous, yellow or greenish discharge. In this stage, the lactobacilli which form the normal vaginal flora is replaced by anaerobic flora similar to that found in bacterial vaginosis. However, asymptomatic women have a normal vaginal $\mathrm{pH}$ with normal vaginal flora. This state of infection is termed the "carrier stage". The clinical signs exacerbate with menstruation and often improve during pregnancy. It is thought that menstrual blood causes a rise in $\mathrm{pH}$ creating an ideal environment for trichomonad reproduction. Also, the elevated iron concentration in menstrual fluid aids in activating various pathogenic mechanisms, such as cysteine proteinases. The hormone estrogen, which levels are decreased during menstruation, has been shown to inhibit a pathological protein known as cell detaching factor. This organism has been associated with 
reproductive tract complications, such as premature rupture of membranes, low birth weight, cervical cancer, infertility, as well as HIV transmission. Trichomoniasis is predominant in women, but men are considered as asymptomatic carriers of this disease. Symptomatic cases, such as urethral discharge, urethritis and prostatitis have been reported in $33 \%$ to $50 \%$ of male trichomonal infections. Also noted is the link between $T$. vaginalis and various complications including pelvic inflammatory disease and infertility.

\section{Diagnosis of Trichomoniasis}

Diagnosis of T. vaginalis's infection is being made on the basis of clinical symptoms, but in women, the characteristics of the vaginal discharge, including color and odor, are poor predictors of $T$. vaginalis. Detection of $T$. vaginalis organism is essential to the diagnosis of $T$. vaginalis because the clinical signs and symptoms are unreliable and as many as $50 \%$ of cases are asymptomatic.

\section{Microscopic Examination}

After a fresh specimen from the vaginal secretion is taken and transferred by a sterile wire loop to a glass slide, a cover slip will be put on it and then be examined using oil immersion high power, dark-field, or phase contrast microscopy. Microscopic examination has been a traditional technique to diagnose trichomoniasis with observation of motile protozoa in vaginal or cervical secretions.

Wet mount examination is straight forward and rapid, but more than $10^{3} / \mathrm{ml}$ of live protozoa are required for detection. Otherwise the sensitivity of direct microscopy varies from as low as $38 \%$ to as high as $82 \%$ depend on the time, experience, the immediate examination of the specimen, and the loss of distinctive motility after the protozoan has been removed from body temperature. The study of Patil M.J. (2012) in India showed the sensitivity and specificity of wet mount, which were $60 \%$ and $100 \%$, respectively, whereas sensitivity and specificity of the In Pouch TV culture system were $73.33 \%$ and $100 \%$, respectively when compared to PCR.

\section{Culture Methods}

The 'gold standard' for the diagnosis of trichomoniasis is broth culture technique using Diamond's medium. The minimum inoculum size required for a positive result is about 10 to 20 organisms $/ \mathrm{ml}$ and the growth of the organism is easy to interpret. However, there are inherent limitations to culture diagnosis. An incubation period of 2 to 7 days is usually necessary to identify $T$. vaginalis in cultures, however during which time infected patients may continue to transmit the infection, and no culture systems are widely available to clinicians. To improve the acceptability of diagnosis by culture, a plastic envelope method was developed, which permits both immediate examination and culture in one self contained system. The results are comparable to those of wet mount and culture techniques. Similar to the plastic envelope method, the InPouch system is a two-chambered bag that allows the performance of an immediate wet-mount by microscopic examination through the bag, as well as a culture. Levi et al. (1997) and Sood et al., (2007) showed that the InPouch system is at least as sensitive as Diamond's modified medium for the detection of $T$. vaginalis. Borchardt et al. (1997)showed that this system is significantly more sensitive than either Diamond's modified medium or Trichosel medium. The cell culture technique uses a variety of cell lines to recover $T$. vaginalis from clinical 
specimens. Garber et al. (1987) used McCoy cells for the cultivation of T.vaginalis from clinical specimens and showed this method to be superior to the broth culture and wetmount preparation since it was able to detect T. vaginalis at a concentration as low as 3 organisms/ml.

\section{Stain Techniques}

Because cultivation methods are relatively slow and wet mount preparation yielded low sensitivity, the staining of parasites in fixed and unfixed smears was introduced.

According to Greenwood J. R. et al. (1981), staining techniques such as acridine orange is almost as sensitive as microscopic examination when specimens can be examined immediately after sampling.

Lowe G.H. (1965) has shown that, examination of a Leishman-stained film yielded the highest proportion of positive results but probably failed to detect trichomonads when their quantity was low. The best combination of methods was found to be Leishman film and culture: the positive yield was $99 \%$. Papanicolaou staining holds considerable appeal in the diagnosis of trichomoniasis because it is routinely used in gynecologic screening for cytologic abnormalities, particularly in populations with a high prevalence of STD. The limited power of culture and microscopic methods for the detection of $T$. vaginalis prompted the advance of the more sophisticated methods which can detect antigen, antibody, or nucleic acids in urethral or vaginal secretion.

\section{Antibody-based Techniques}

Various techniques including agglutination, complement fixation, indirect hemagglutination, gel diffusion, fluorescent antibody, and enzyme-linked immunosorbent assay have been used to demonstrate the presence of antitrichomonal antibodie. Mason P.S. et al. (2001) reported that, enzyme immunoassay (EIA) was used to detect antibodies to Trichomonas vaginalis in sera from Zimbabwe. The EIA showed a sensitivity of 94 to $95 \%$ when compared with vaginal swab culture. The specificity was 77 to $85 \%$ in the two groups. The EIA may be useful for community surveys of trichomoniasis. Because $T$. vaginalis is a common sexually transmitted disease, the test may indicate behavior that increases the risk of STD transmission.

Fig.A Morphology of Trichomonas Vaginalis

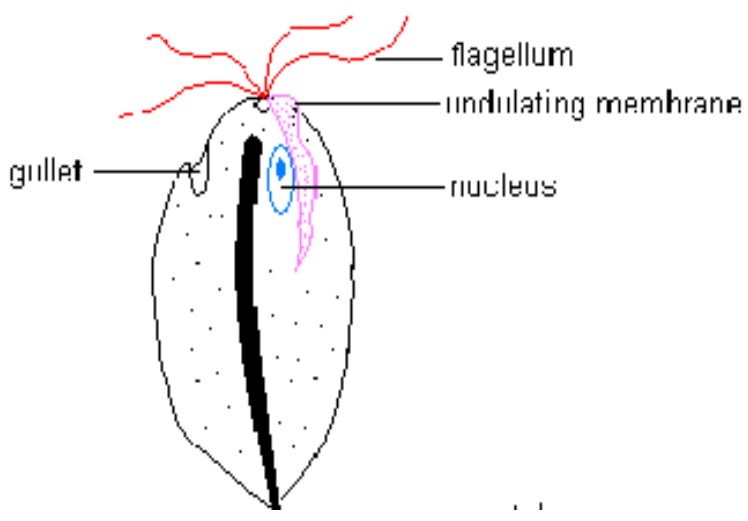


Fig.B Life cycle of Trichomonas vaginalis

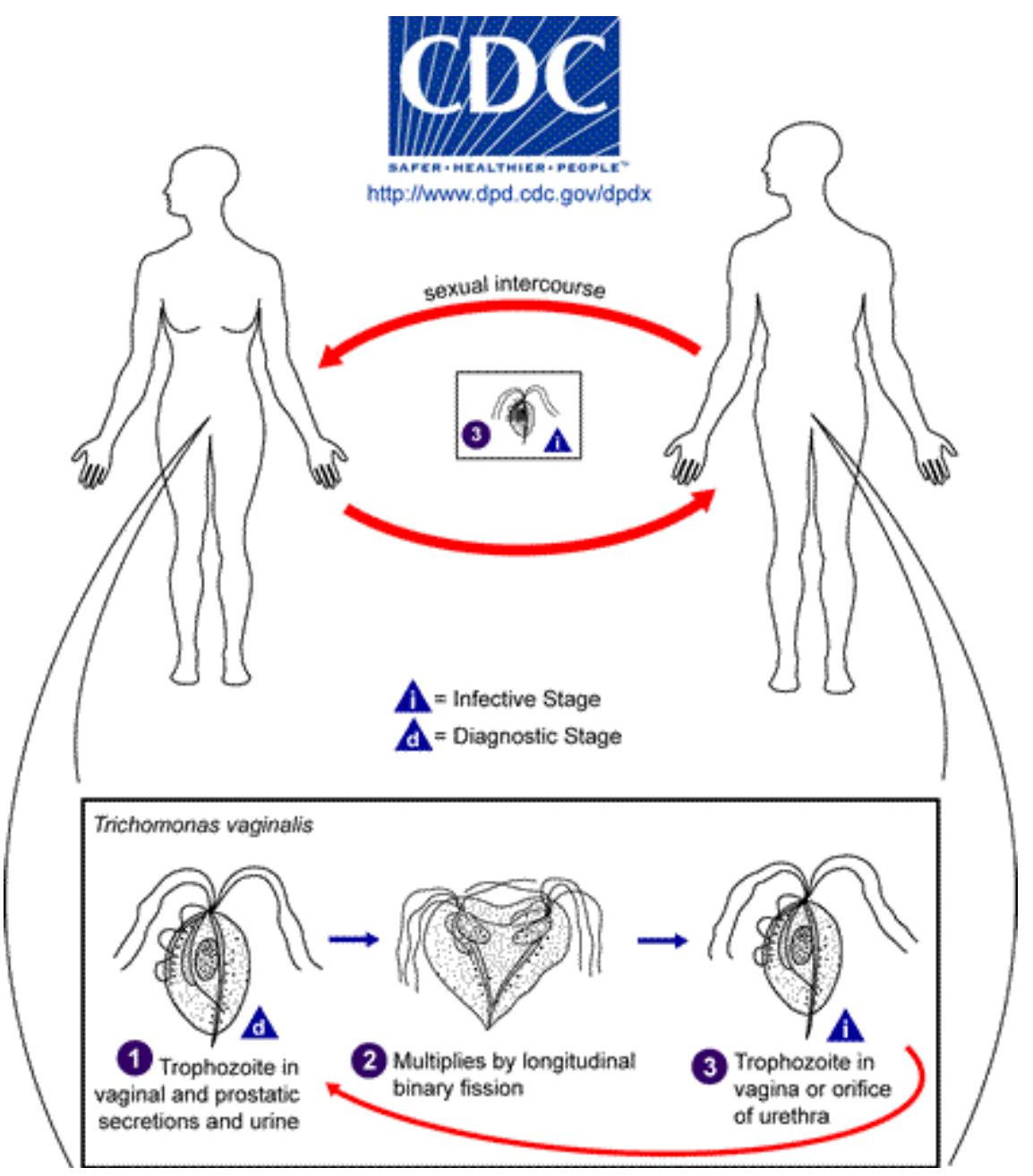

\section{Molecular Techniques}

Recombinant DNA techniques have been increasingly used in clinical laboratories to improve the specificity and sensitivity of $T$. vaginalis diagnosis. Many studies reported that, PCR appears to be the method of choice for the detection of genital $T$. vaginalis infections with high sensitivities and excellent specificities for both vaginal samples and male urethral samples.

\section{Treatment}

Metronidazole $2 \mathrm{~g}$ orally in a single dose or Tinidazole $2 \mathrm{~g}$ orally in a single dose
Alternate regimen: Metronidazole $500 \mathrm{mg}$ twice a day for 7 days

Pregnancy (CDC-recommended regimen) Metronidazole $2 \mathrm{~g}$ orally in a single dose All symptomatic pregnant women should be treated, regardless of pregnancy stage.

\section{References}

Ackers, J.P. Immunologic aspects of human trichomoniasis. In B. M. Honigberg (ed.),Trichomonads Parasitic in Humans, Springer-Verlag, New York.1990; pp. 36-52.

Alderete, J.F., \& Garza, G.E. Specific nature of Trichomonas vaginalis parasitism 
of host cell surfaces. Infection and Immunity. 1985; 50:701-708.

Alderete, J.F., \& Provenzano, D. The vagina has reducing environment sufficient for activation of Trichomonas vaginalis cysteine proteinases. Genitourinary Medicine,73:291-296. Sexually Transmitted Diseases. 1997; 17:106-109.

Alderete, J.F., Benchimol, M., Lehker, M.W., \& Crouch, M.L. The complex fibronectin-Trichomonas vaginalis interactions and Trichomoniasis. Parasitology International.2002; 51:285-292.

Arroyo, R., Gonzalez-Robles, A., MartinezPalomo A \& Alderete, J.F. 1993. Signaling of Trichomonas vaginalis for amoeboid transformation and adhesion synthesis follows cytoadherence. Molecular Microbiology, 7:299-309.

Beal, C., Goldsmith R, Kotby M, Sherif M, el-Tagi A, Farid A, Zakaria S, and Eapen J,The plastic envelope method, a simplified technique for culture diagnosis of trichomoniasis. J. Clin. Microbiol. 1992. 30: 22652268.

Bhalla P, Chawla R, Garg S, Singh MM, Raina U, Bhalla R, et al.Prevalence of a bacterial vaginosis among women in Delhi, India. Indian J Med Res 2007; 125:167-72.

Borchardt, K.A., Zhang MZ, Shing H, Flink $\mathrm{K}$, A comparison of the sensitivity of the InPouch TV, Diamond's, and Trichosel media for detection of Trichomonas vaginalis,Genitourin Med 1997;73:297-298.

Centers for Disease Control and Prevention, CDC. Division of STD Prevention:www.cdc.gov/std.2010 CDC STD treatment guidelines.

Cepika, I., Hampl, V., Kulda, J., \& Flegr, J.New evolutionary lineages, unexpected iversity, and host specificity in the parabasalid genus Tetratrichomonas.

Molecular Phylogenetics and Evolution.2006; 39:542-551.

Chen, W.L., Chen, J.F., Zhong, X.R., Liang, P., \& Lin, W. Ultrastructural and immunohistochemical studies on Trichomonas vaginalis adhering to and phagocytizing genitourinary cells. Chinese Medical Journal (Engl)2004; 117:376-381.

Daly, J.J., Sherman, J.K., Haley, T., \& Hostetler, T.L. Difference in effect of dog seminal fluid and human seminal fluid and semen on in vitro survival of Trichomonas vaginalis.Sex Transm Dis. 1990;17(2):106-9.

Draper, D., Donohoe, W., Mortimer, L., \& Heine, R.P.Cysteine proteases of Trichomonas vaginalis degrade secretory leukocyte protease inhibitor. Journal of Infectious Diseases.1998; 178:815-819.

Fouts, A.C., \& Kraus, S.J. Trichomonas vaginalis: reevaluation of its clinical presentation and laboratory diagnosis. Journal of Infectious Diseases.1980; 141:137-143.

Fouts, A.C., and Kraus SJ, Trichomonas vaginalis: reevaluation of its clinical presentation and laboratory diagnosis. J. Infect. Dis.1980. 141:137- 143 .

Frohlich, J.A., Karim, Q.A., Mashego, M.M., Sturm, A.W., \& Karim, S.S.A.Opportunities for treating sexually transmitted infections and reducing HIV risk in rural South Africa. Journal of Advanced Nursing.2007; 60(4):377-383.

Garber, G.E., Lemchuk-Favel, L.T., \& Rousseau, G. Effect of betaestradiol production of the celldetaching factor of Trichomonas 
vaginalis. Journal of Clinical Microbiology.1991; 29:1847-1849.

Garber, G.E., Sibau L, Ma R, Proctor EM, Shaw CE, Bowie WR, Cell Culture Compared with Broth for Detection of Trichomonas vaginalis, J Clin Microbiol. 1987 Jul;25(7):1275-9.

Germot, A., \& Philippe, H.Critical analysis of eukaryotic phylogeny: a case study based on the HSP70 family. Journal of Eukaryotic Microbiology.1999; 46:116-124.

Goode, M.A., Grauer, K., \& Gums, J.G. Infectious vaginitis: selecting therapy and preventing recurrence. Postgraduate Medicine.1994; 96:85-98.

Greenwood, J.R., Kirk-Hillaire K, Evaluation of Acridine Orange Stain for Detection of Trichomonas vaginalis in Vaginal Specimens, $J$ Clin Microbiol. 1981 Dec;14(6):699.

Hashimoto, T., Sanchez, L.B., Shirakura, T., Muller, M., \& Hasegawa, M. Secondary absence of mitochondria in Giardia lamblia and Trichomonas vaginalis revealed by valyl-tRNA synthetase phylogeny. Proceedings of the National Academy of Sciences of the United States of America1998; 95:6860-6865.

Heine, P., \& McGregor, J.A. Trichomonas vaginalis: a reemerging pathogen. Clinical Obstetrics and Gynecology.1993; 36:137-144.

Hesseltine, H., Wolters, S.L., \& Cambell, A. Experimental human vaginal trichomoniasis. Journal of Infectious Diseases. 1942; 71:127-130.

Johnson, L.F., Coetzee, D.J., \& Dorrington, R.E. Sentinel surveillance of sexually transmitted infections in South Africa: a review.2005 Sexually Transmitted Infections,81:287-293.

Krieger, J.N. 1995. Trichomoniasis in men: old issues and new data.
SexuallyTransmitted Diseases, 22:83-96.

Krieger, J.N. Trichomoniasis in men: old issues and new data. Sexually Transmitted Diseases.1995; 22:8396.

Lanceley, F., \& McEntegart, M.G. Trichomonas vaginalis in the male; the experimental infection of a few volunteers. Lancet.1953; 1:668-671.

Lehker, M.W., \& Sweeney, D. Trichomonad invasion of the mucous layer requires adhesins, mucinases, and motility. Sexually Transmitted Infections. 1999; 75:231-238.

Levi, M.H., Torres J, Pina C, Klein RS. Comparison of the InPouch TV culture system and Diamond's modified medium for detection of Trichomonas vaginalis. $J$ Clin Microbiol 1997; 35 : 3308-10.

Lossick, J.G. Epidemiology of urogenital trichomoniasis. In Honigberg $\mathrm{B}$. M.(ed.).Trichomonads parasitic in humans. Springer-Verlag, New York.1990a; pp. 311-323.

Lowe, G.H., A comparison of current laboratory methods and a new semisolid culture medium for the detection of Trichomonas vaginalis, J Clin Pathol. 1965 Jul;18:432-4.

Madhivanan P, Krupp K, Chandrasekaran V, Karat C, Arun A, Cohen CR, Reingold AL,Klausner JD. Prevalence and correlates of bacterial vaginosis among young women of reproductive age in Mysore, India. Indian J Med Microbial 2008; 26:132-7.

Martin, R.D., Kaufman, R.H., and Burns, M, Trichomonas vaginalis: a statistical evaluation of diagnostic methods. Am. J. Obstet. Gynecol. 1963. 87:1024-102.

Mason, P.R., Gregson, S., Gwanzura L, Cappuccinelli P, Rapelli P, Fiori PL, 
Enzyme immunoassay for urogenital trichomoniasis as a marker of unsafe sexual behavior, Epidemiol. Infect., 126, 103-109.

McCann, J.S. 1974. Comparison of direct microscopy and culture in the diagnosis of trichomoniasis. Br. J. Vener. Dis. 50:450-452.

Moldwin, R.M., Sexually transmitted protozoal infections. Trichomonas vaginalis,Entamoeba histolytica, and Giardia lamblia. Urol. Clin. North Am. 1992. 9:93-101.

Moodley, P., \& Sturm, A.W. Management of vaginal discharge syndrome: how effective is our strategy? International Journal of Antimicrobial Agents.2004; 24S:S4S7

Moodley, P., \& Sturm, A.W. Sexually transmitted infections, adverse pregnancy outcome and neonatal infection. Seminars in Neonatology.2000; 5:255-269.

Palmer, J.D. Organelle genomes: going, going, gone! Science.1997; 275:790791.

Patil, M.J., Nagamoti JM, Metgud SC, Diagnosis of Trichomonas vaginalis from Vaginal Specimens by Wet Mount Microscopy, In Pouch TV Culture System, and PCR, J Glob Infect Dis. 2012 Jan;4(1):22-5.

Petrin, D., Delgaty K., Bhatt, R., \& Garber, G.C .Clinical and microbiological aspects of Trichomonas vaginalis. Clinical Microbiology Reviews.1998;11:300-317.

Petrin, D., Delgaty, K., Bhatt, R., \& Garber, G.C. Clinical and microbiological aspects of Trichomonas vaginalis. Clinical Microbiology Reviews.1998; 11:300-317.

Pindak, F.F., Gardner WA Jr, Mora de Pindak M, Growth and cytopathogenicity of Trichomonas vaginalis in tissue cultures. J. Clin. Microbiol. 1986. 23:672-678

Popescu, C.F., Bădulescu A, Bădulescu F, Cotarcea S, Găvănescu $\mathrm{M}$, Preliminary study concerning the Cytoscreen system importance (Liquid Based Cytology) in gynecologic cytology, Romanian J. Morphology and Embryology 2005, 46(1):23-27.

Rein, M.F.Clinical manifestations of urogenital trichomoniasis in women. In Honigberg B.M (ed.) Trichomonads Parasitic in Humans.1989; Springer-Verlag,

Ryan-Wenger, N.A., Neal JL, Jones AS, Lowe NK, Accuracy of vaginal symptom selfdiagnosis algorithms for deployed military women, Nurs Res. 2010 Jan-Feb; 59(1):2-10

Ryu, J.S., \& Min, D.Y. Trichomonas vaginalis and trichomoniasis in the Republic of Korea. Korean Journal of Parasitology.2006; 44:101-116.

Shaio, M.F., \& Lin, P. Influence of humoral immunity on leukotriene B4 production by neutrophils in response to Trichomonas vaginalis stimulation. Parasite Immunology. 1995;17:127-133.

Sibau, L., Bebb D, Proctor EM, Bowie WR, Enzyme-linked immunosorbent assay for the diagnosis of trichomoniasis in women. Sex. Transm. Dis. 1987. 14:216-220.

Sood, S., Mohanty S, Kapil A, Tolosa J, Mittal S, InPouch TV culture for detection of Trichomonas vaginalis, Indian J Med Res 125, April 2007, pp 567-571.

Spence MR, Hollander DH, Smith J, McCaig L, Sewell D, Brockman M, The clinical and laboratory diagnosis of Trichomonas vaginalis infection. Sex. Transm. Dis. 7:168-171.

Stary, A., Kuchinka-Koch A, Teodorowicz 
L, Detection of Trichomonas vaginalis on modified Columbia agar in the routine laboratory. $\mathrm{J}$ Clin Microbiol 2002;40:3277-80.

Sturm, A.W., Wilkinson, D., Ndovela, N., Bowen, S., \& Connolly, C. Pregnant women as a reservoir of undetected sexually transmitted diseases in rural South Africa:implications for disease control. American Journal of Public Health.1998; 88:1243-1245.

Thorburn, A.L. Alfred Francois Donne, 1801-1878, discoverer of Trichomonas vaginalis and of leukaemia. British J. Veneral Disease. 1974; 50:377-380.

Upcroft, P., \& Upcroft, J.A.Drug targets and mechanisms of resistance in the anaerobic protozoa. Clinical Microbiological Reviews.2001; 14(1):150-164.

Valadkhani, Z., Sharma, S., Harjai, K., Gupta, I., \& Malla, N. 2003. In vitro comparative kinetics of adhesive and haemolytic potential of $T$. vaginalis isolates from symptomatic and asymptomatic females. Indian Journal of Pathological Microbiology, 46:693-699.

van Der Schee, C., van Belkum A, Zwijgers $\mathrm{L}$, et al. 1999. Improved Diagnosis of Trichomonas vaginalis Infection by PCR Using Vaginal Swabs and Urine Specimens Compared to Diagnosis by Wet Mount Microscopy, Culture, and Fluorescent Staining, $J$ Clin Microbiol., 37(12): 4127-30.

Wisdom, A.R., \& Dunlop, E.M. Trichomoniasis: study of the disease and its treatment in women and men. British Journal of Veneral Disease.1965; 41:90-96.

Wolner-Hanssen, P., Krieger, J.N., Stevens, C.E., Kiviat, N.B., Koutsky, L., DeRouen,T.,Hillier, S., \& Holmes, K.K. Clinical manifestations of vaginal trichomoniasis. Journal of the American Medical Association 1989; 261:571-576.

World Health Organization. 2001. Global prevalence and incidence of selected curable sexually transmitted infections: overview and estimates. World Health Organization Geneva, pp. 1-43.

Zariffard, M.R., Harwani, S., Novak, R.M., Graham, P.J., Ji, X., \& Spear, G.T.Trichomonas vaginalis infection activates cells through toll-like receptor 4. Clin. Immunol., 111: 103107. New York. 2004; pp.225-234.

\section{How to cite this article:}

Hodiwala Bhesania, A., and Narayankhedkar, A. 2016. Trichomoniasis-A Review. Int.J.Curr.Microbiol.App.Sci. 5(6): 731-741. doi: http://dx.doi.org/10.20546/ijcmas.2016.506.079 